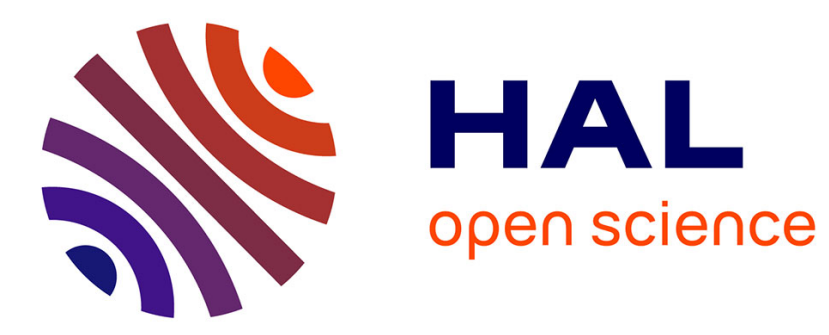

\title{
Raman frequency shift induced by photorefractive effect on Fe-doped lithium niobate
}

\author{
M Bazzan, Ninel Kokanyan, A Zaltron, N Argiolas, D. Chapron, M. D.
} Fontana

\section{- To cite this version:}

M Bazzan, Ninel Kokanyan, A Zaltron, N Argiolas, D. Chapron, et al.. Raman frequency shift induced by photorefractive effect on Fe-doped lithium niobate. Journal of Applied Physics, 2013, 114 (16), pp.163506. 10.1063/1.4826628 . hal-01385633

\section{HAL Id: hal-01385633 \\ https://hal.science/hal-01385633}

Submitted on 21 Oct 2016

HAL is a multi-disciplinary open access archive for the deposit and dissemination of scientific research documents, whether they are published or not. The documents may come from teaching and research institutions in France or abroad, or from public or private research centers.
L'archive ouverte pluridisciplinaire HAL, est destinée au dépôt et à la diffusion de documents scientifiques de niveau recherche, publiés ou non, émanant des établissements d'enseignement et de recherche français ou étrangers, des laboratoires publics ou privés. 


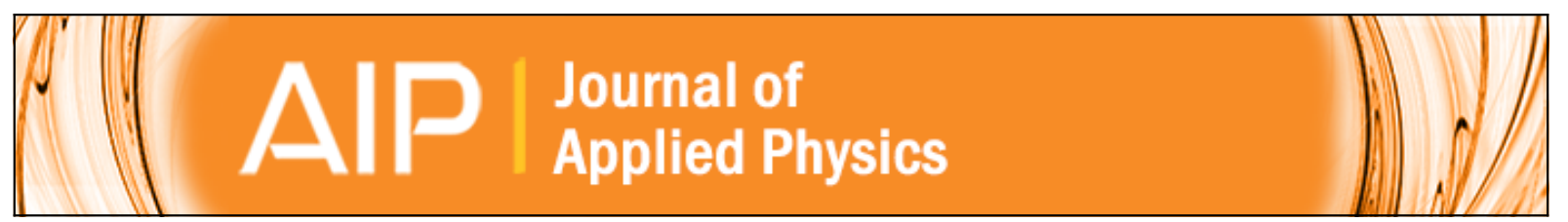

Raman frequency shift induced by photorefractive effect on Fe-doped lithium niobate

M. Bazzan, N. Kokanyan, A. Zaltron, N. Argiolas, D. Chapron, and M. D. Fontana

Citation: Journal of Applied Physics 114, 163506 (2013); doi: 10.1063/1.4826628

View online: http://dx.doi.org/10.1063/1.4826628

View Table of Contents: http://scitation.aip.org/content/aip/journal/jap/114/16?ver=pdfcov

Published by the AIP Publishing

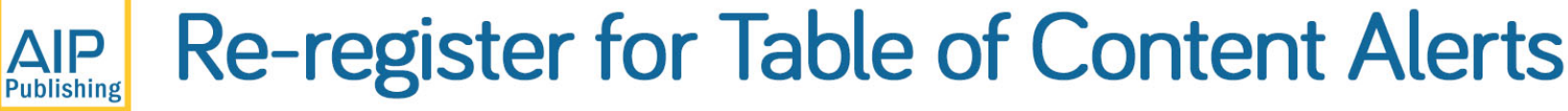

Create a profile.

Sign up today! 


\title{
Raman frequency shift induced by photorefractive effect on Fe-doped lithium niobate
}

\author{
M. Bazzan, ${ }^{1}$ N. Kokanyan, ${ }^{2,3}$ A. Zaltron, ${ }^{1}$ N. Argiolas, ${ }^{1}$ D. Chapron, ${ }^{2,3}$ and M. D. Fontana ${ }^{2,3}$ \\ ${ }^{1}$ Physics and Astronomy Department, Università di Padova, Via Marzolo 8, 35131 Padova, Italy \\ ${ }^{2}$ Université de Lorraine, Laboratoire Matériaux Optiques, Photonique et Systèmes, 2 Rue E. Belin, \\ 57070 Metz, France \\ ${ }^{3}$ Supélec, Laboratoire Matériaux Optiques, Photonique et Systèmes, 2 Rue E. Belin, 57070 Metz, France
}

(Received 15 August 2013; accepted 7 October 2013; published online 24 October 2013)

\begin{abstract}
We report on the observation of a time dependent frequency shift of the Raman modes in photorefractive $\mathrm{Fe}: \mathrm{LiNbO}_{3}$. The origin of this shift is attributed primarily to the space charge field which builds up due to photo-induced charge transport by the excitation beam, and to the subsequent piezo-induced strain. This phenomenon can be regarded as the Raman analogous of the photorefractive effect, in which the space charge field is detected by an electro-optic variation of the refractive index. It should be possible therefore to analyze it using standard models for photorefractivity. In this paper, we verify this claim discussing its validity. (C) 2013 AIP Publishing LLC. [http://dx.doi.org/10.1063/1.4826628]
\end{abstract}

\section{INTRODUCTION}

Raman analysis is a powerful spectroscopic technique, which can be used for the physico-chemical characterization of a number of different materials. Modern Raman spectrometers are often used in conjunction with advanced confocal microscopes, coupling the power of spectroscopic analysis with micron-sized resolution and making it a complete micro-analytical technique, which is largely employed to study technologically important materials. ${ }^{1-3}$

This is the case of lithium niobate (LN), a key material in photonic technology characterized by a combination of pronounced functional properties, which is currently used in a number of different applications such as electro-optics, acousto-optics, and frequency conversion. ${ }^{4,5}$ Lithium niobate shows another remarkable property, i.e., photorefractivity, a semi-permanent modulation of the material's refractive index induced by an inhomogeneous illumination. The photorefractive (PR) effect is due to photo-induced charge transport phenomena, which may lead to a redistribution of mobile charges inside the sample. In turn, this charge separation originates an intense internal electric field, which modulates the refractive index via the electro optic effect. ${ }^{6}$

Nippus and Claus ${ }^{7}$ showed that photorefractivity can affect a Raman spectrum in lithium niobate, especially in samples doped with photorefractive-enhancing ions. Under proper conditions, they showed a decrease in the intensity of Raman lines due to the photorefractive defocusing of the excitation beam inside the material. More recently, Mouras et al. have reported on the activation of forbidden lines in a Raman spectrum, which was attributed to a partial light depolarization effect caused by photorefractivity in $\mathrm{LN}: \mathrm{Fe} .{ }^{8}$ In the present paper, we report on another effect due to photorefractivity, i.e., a time-dependent shift in the frequency of Raman lines. This aspect can significantly affect the result of an experiment focused on the absolute measurement of the Raman line frequency of a sample with strong PR sensitivity. On the other hand, this phenomenon could possibly be used to characterize the photorefractive properties of a sample in a simple way and with the advantage of the high spatial resolution offered by a confocal Raman apparatus. In the following, the phenomenon will be presented and its interpretation in terms of a piezo-induced distortion originating from the photorefractive space charge field will be given. Subsequently, the conditions for the observation of the phenomenon and the possibility to use it as a characterization tool will be discussed.

\section{EXPERIMENTAL}

We measured four different Fe:LN samples grown by the Czochralski method and prepared at the crystal growth laboratory of the University of Padua. Three samples were cut from a boule grown from a $0.1 \%$ mol. Fe:LN melt. The fourth sample was obtained from a boule grown from a $0.05 \%$ mol. Fe:LN melt. All the samples have dimensions of about $10 \times 10 \times 1 \mathrm{~mm}^{3}$ with the major surface optically polished and oriented along the X-direction. Two of the samples with $0.1 \% \mathrm{~mol}$. in the melt were thermally treated in order to modify their reduction degree. Finally, all the samples were characterized by optical absorption in order to measure separately the effective concentration of $\mathrm{Fe}^{2+}$ and $\mathrm{Fe}^{3+}$ according to the method detailed in Ref. 9. The results are reported in Table I.

All the experiments were performed using a Horiba ARAMIS confocal micro Raman spectrometer at the Lorraine University. The excitation line of $633 \mathrm{~nm}$ and the backscattered radiation were polarized and analyzed, respectively, using two linear polarizers either parallel or perpendicular to the c-axis of the sample. Raman spectra in configuration $\mathrm{X}(\mathrm{zz}) \mathrm{X}$ (modes $\mathrm{A}_{1}[\mathrm{TO}]$, a typical spectrum is given in Figure 1) and $\mathrm{X}(\mathrm{yz}) \mathrm{X}$ (modes E[TO]) were collected and analyzed. ${ }^{10}$

A typical measurement run consists in acquiring a series of Raman spectra on a given sample in successive moments. The time required for recording a single Raman spectrum 
TABLE I. Summary of sample codes and characteristics.

\begin{tabular}{lcccc}
\hline \hline & \multicolumn{4}{c}{ Fe } \\
$\begin{array}{l}\text { Sample code } \\
\text { and description }\end{array}$ & $\begin{array}{c}\text { concentration } \\
(\% \text { mol. })\end{array}$ & $\begin{array}{c}{\left[\mathrm{Fe}^{2+}\right]} \\
\left(\times 10^{24} \text { at. } / \mathrm{m}^{3}\right)\end{array}$ & $\begin{array}{c}{\left[\mathrm{Fe}^{3+}\right]} \\
\left(\times 10^{24} \text { at. } / \mathrm{m}^{3}\right)\end{array}$ & {$\left[\mathrm{Fe}^{2+}\right] /\left[\mathrm{Fe}^{3+}\right]$} \\
\hline $\begin{array}{l}\text { Fe:LN 0.1 O } \\
\text { (oxidized) }\end{array}$ & 0.1 & 0 & 18.8 & $<0.01$ \\
$\begin{array}{l}\text { Fe:LN 0.1 AG } \\
\text { (as-grown) }\end{array}$ & 0.1 & 0.46 & 18.34 & $0.025 \pm 0.008$ \\
$\begin{array}{l}\text { Fe:LN 0.1 R } \\
\text { (reduced) }\end{array}$ & 0.1 & 5.29 & 13.5 & $0.39 \pm 0.01$ \\
\begin{tabular}{l} 
Fe:LN 0.05 \\
\hline \hline
\end{tabular} & 0.05 & 1.57 & 7.83 & $0.20 \pm 0.01$ \\
\hline
\end{tabular}

depends on the intensity of the measured signal, determined in turn by the power of the incident beam and on the confocal pinhole aperture: in our case, it was between 0.2 and $5 \mathrm{~s}$. The time step between two successive acquisitions was set between 1 and $5 \mathrm{~s}$, depending on the rapidity of the sample response. The peak frequency, width, and integrated intensity of each Raman line were fitted by an automatic routine using a damped oscillator model and the results were plotted as a function of time.

The fundamental condition to observe the phenomena mentioned below is that the acquisition time of a Raman specrum is significantly shorter than the photorefractive time constant of the material. In a given sample, the speed of the PR phenomenon is influenced primarily by the intensity of the illumination pattern, so that it is mandatory to use low intensities to have large time constants; however, in this way, the signal to noise ratio is reduced forcing the acquisition time of each Raman spectrum to be longer, so that a trade-off is present. It is possible to relax these experimental requirements by using an objective with low magnification so that the power density on the sample is reduced without affecting the signal to noise ratio of the Raman spectra. Also the confocal aperture plays an important role. In order to obtain fast acquisition times, we had to use a large pinhole size (fixed at $400 \mu \mathrm{m}$ ) and consequently large detection volume; this has important consequences in the dynamics of the

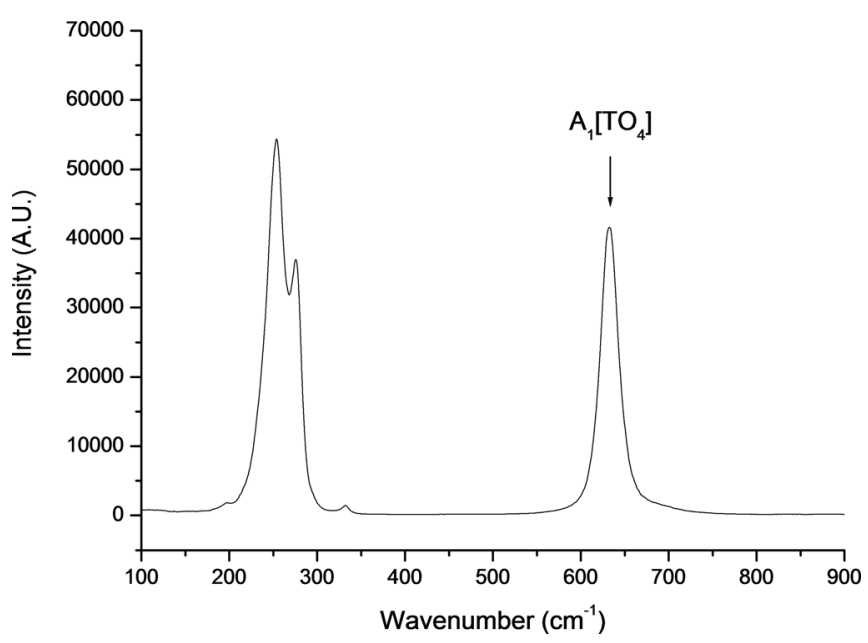

FIG. 1. Typical Raman spectrum showing the $\mathrm{A}_{1}[\mathrm{TO}]$ modes, obtained in the $\mathrm{X}(\mathrm{zz}) \mathrm{X}$ configuration using an excitation line at $632.8 \mathrm{~nm}$. The $\mathrm{A}_{1}\left[\mathrm{TO}_{4}\right]$ mode is indicated. measured signal, as it will be shown below. In the following, we used a $10 \times$ objective and an excitation beam with a wavelength of $632.8 \mathrm{~nm}$; the resulting focused spot size had a waist radius at half of the maximum intensity of $15 \mu \mathrm{m}$. The incident power was varied by means of three neutral density filters and measured before the sample surface with the help of a portable power meter to be in the range between 10 and $1 \mathrm{~mW}$. The average intensity on the illuminated spot is estimated by dividing the beam power by the waist area and correcting it for Fresnel reflections.

In our experiments, we aim at investigating the effect of a light-induced quasi static space charge field on the Raman spectra. This study can be compared with the changes in the Raman spectra directly induced by a static electric field or a temperature change applied on the sample. Therefore, we performed also a series of experiments that were useful to clarify our observations. The effect of a static electric field on the Raman spectra was evaluated in the following way: a couple of planar electrodes were deposited on the $\mathrm{X}$ surface of an undoped LN sample, leaving between them a gap of about $100 \mu \mathrm{m}$, so that the electric field was mainly along the $\mathrm{Z}$ direction. Those electrodes were connected with a power supply able to span a voltage range of $1 \mathrm{kV}$, which can be doubled by inverting the electrical connections with the sample. We measured the Raman spectra at different voltages focusing at the surface of the region between the electrodes, using the lowest possible power and an excitation wavelength at $785 \mathrm{~nm}$, in order to limit to the maximum possible extent the influence of the photorefractive effect.

Moreover, the PR effect on the Raman spectrum has to be distinguished from other contributions, such as those deriving from sample heating, which may play a role during the Raman measurements. Then, in order to verify whether the heating of the sample due to the sample absorption can affect the Raman modes frequency, we performed an independent measurement of the Raman frequency change due to the sample temperature with a $785 \mathrm{~nm}$ excitation laser. A pure lithium niobate sample (i.e., with negligible absorption) was therefore mounted into a thermal stage and measured between room temperature and $350 \mathrm{~K}$.

\section{RESULTS}

By observing the time evolution of the Raman spectra, we found that, in general, all the Raman peak frequencies shift with the time. Moreover, an overall decrease of the spectrum intensity, which may be reduced down to two thirds of the initial value in the highly doped sample, is also observed, as expected from Ref. 7. Although all the modes are shifted of a comparable amount, the phenomenon is particularly evident for the $\mathrm{A}_{1}\left[\mathrm{TO}_{4}\right]$ mode because this line is intense, well separated and with a relatively large Raman shift of $\sim 632 \mathrm{~cm}^{-1}$ (see Figure 1), which makes it particularly sensitive to any perturbation of the lattice structure, so that in the following, we will concentrate on this specific line. In Figure 2, it is reported the time evolution of the $\mathrm{A}_{1}\left[\mathrm{TO}_{4}\right]$ frequency shift for samples Fe:LN 0.05 and Fe:LN 0.1-AG (see Table I for sample description). The shift evolves according to a saturation behavior and reaches a 


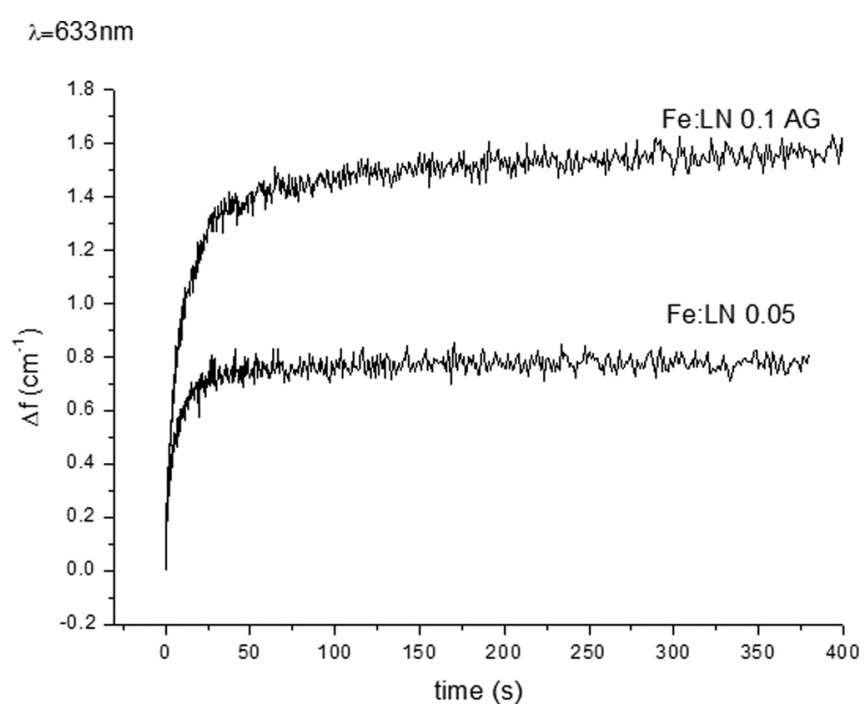

FIG. 2. Time evolution for the peak frequency of the $\mathrm{A}_{1}\left[\mathrm{TO}_{4}\right]$ mode in samples Fe:LN 0.05 and Fe:LN 0.1 AG (see Table I for sample description).

limit value as high as $0.7 \mathrm{~cm}^{-1}$ and $1.4 \mathrm{~cm}^{-1}$ for sample Fe:LN 0.05 and Fe:LN 0.1-AG, respectively. As anticipated, the time evolution of the frequency shift depends on the light intensity, becoming faster for higher intensities.

Separate experiments were performed to obtain the peculiar dependences of Raman line positions on controlled external electric field and temperature. When the field is ramped between $-10^{6}$ to $10^{6} \mathrm{~V} / \mathrm{m}$, a linear change in the frequency of the Raman modes is observed, in agreement with Ref. 11. The sign of the frequency change is the same as the light-induced shift and the magnitude is comparable. By repeating several times our experiments and by fitting the obtained dependences with a linear law we were able to estimate a calibration coefficient of $\sim 10^{-7} \mathrm{~cm}^{-1} \mathrm{~m} / \mathrm{V}$ for the $\mathrm{A}_{1}\left[\mathrm{TO}_{4}\right]$ mode.

The experiments as function of temperature show an opposite behavior since in the explored range the frequency is shifted to lower values by increasing the temperature. From our data, we obtain a calibration coefficient of $-0.024 \mathrm{~cm}^{-1} / \mathrm{K}$. The observed shift values are quite small if compared with the typical accuracy of the employed spectrometer, which is $\sim 0.6 \mathrm{~cm}^{-1}$. However, the latter value accounts for both random and systematic errors in the determination of a single, ideally narrow spectral component. In our case, the Raman peaks are composed by a large number of data points, and the peak frequency is determined by fitting them with a Lorentz function so that the random component of the uncertainty on the peak position (due essentially to the discretization of the CCD pixels and to electronic noise) is strongly reduced. On the other hand, the systematic part of the uncertainty (which in a single measurement is due essentially to the drift of the spectrometer response with time) plays practically no role as far as the spectrometer drift is slow on the timescale of a single measure. For these reasons, we believe that the effective precision and accuracy of our frequency shift measurements are better than the nominal ones and could be estimated to be about $0.1 \mathrm{~cm}^{-1}$ from the SNR of Figure 2 and the RMS errors of our repeated measurements.

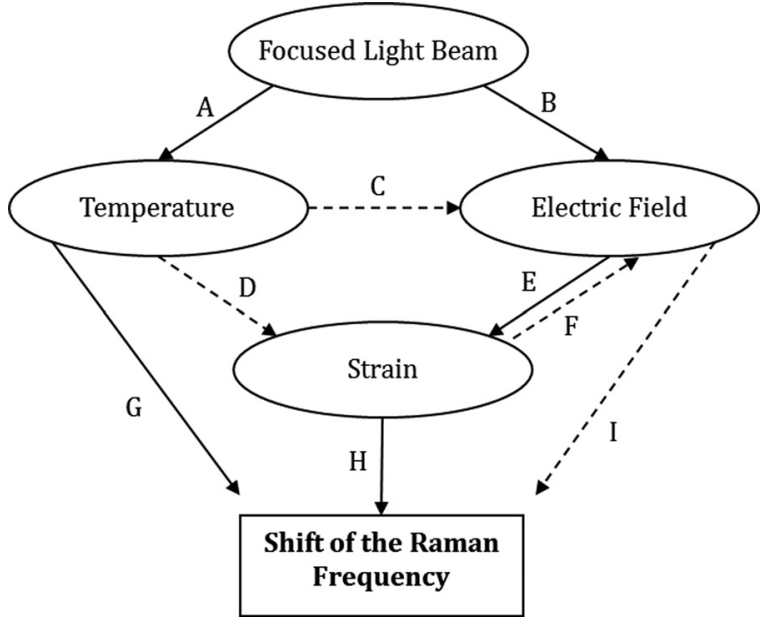

FIG. 3. Scheme of the possible couplings between different light induced effects in Fe:LN. Dashed arrows represent contributions that can be neglected.

\section{DISCUSSION AND ANALYSIS}

\section{A. Physical origin of the observed shifts}

It is clear that the time dependent shift of the Raman lines is due to some kind of local modification induced by the excitation beam in the sample. A change in Raman lines frequency can be due to several reasons, such as a change in the sample temperature or stress. Moreover, lithium niobate is a polar crystal exhibiting a strong coupling among different thermodynamic fields, so that we have to consider also secondary effects. Our first aim is therefore to figure out what are the important couplings responsible for the observed shifts in the Raman spectra.

In Figure 3, are represented the possible effects arising when an absorbing lithium niobate sample is illuminated with a tightly focused laser beam, together with the possible couplings between the different fields. The arrow $B$, the photoinduced charge transport, is of particular interest to us. It is known ${ }^{12}$ that typical space charge fields in $\mathrm{Fe}: \mathrm{LiNbO}_{3}$ samples can easily reach values as high as $\sim 10^{7} \mathrm{~V} / \mathrm{m}$, with a major component along the positive $\mathrm{z}$ direction, due to the particular form of the photogalvanic tensor of the material. Our experiments under static applied field prove that a field of this magnitude is sufficient to give a frequency shift comparable to the observed ones and with the correct sign.

At first, we examine the possibility that the space charge field may shift the frequencies of the modes by some direct influence on the Raman effect, disregarding for the moment the influence of other fields. Classically, the Raman effect can be understood considering that the high frequency dielectric polarizability can be modified by a phonon mode, described by a generalized coordinate associated to the displacement of the ions inside the crystal cells. The derivatives of the polarizability with respect to the phononic generalized coordinates constitute the entries of the so-called Raman tensor. With reference to Figure 3, we consider now the possibility that an electric field can somehow change directly the Raman tensor itself (path B-I in Figure 3). This would imply to consider an additional derivative in the expansion 
of the polarizability (hyperpolarizability). Such an effect shall probably affect the intensity of the Raman lines (which is proportional to the respective component of the Raman tensor) rather than their frequency, which is determined by the lattice vibrational dynamics and therefore probably not directly influenced by an electric field. Moreover, in this case, we would be dealing with a third-order effect, and therefore probably very small compared to other couplings represented in Figure 3.

Excluded a direct influence of the space charge field, we look now for indirect couplings. It is known that strain may modify the frequencies of Raman modes. As LN is piezoelectric, the photorefractive electric field is able to induce such a strain, affecting indirectly the lattice modes frequency (path B-E-H in Figure 3). The possibility of inducing a measurable piezoelectric strain in lithium niobate by applying an external field of the same order of magnitude of the photorefractive, one has already been reported by in situ X-ray studies. ${ }^{13}$ Moreover, in semiconductor materials, it is known that such strain fields produce frequency shifts of the same order of magnitude of the ones here reported. ${ }^{14}$ It is therefore reasonable to consider the photorefractive-piezoelectric coupling as a possible cause of the observed shifts.

The second possibility which may lead to a shift of the frequency comes from thermal effects. The local heating of the sample (arrow A in Figure 2) has been studied numerically using the $\mathrm{COMSOL}^{\circledR}$ multiphysics simulation software. The illumination with the excitation beam is modeled with a focused Gaussian beam with a waist of $15 \mu \mathrm{m}$ and a power of $10 \mathrm{~mW}$. We considered a lithium niobate sample with a typical absorption coefficient of $20 \mathrm{~cm}^{-1}$, which corresponds to our most absorbing case (sample Fe:LN 0.1-red). In this case, the maximum heating in stationary conditions resulted in an overall temperature increment of $\sim 10 \mathrm{~K}$, assuming an emissivity of $0.9,{ }^{15}$ and a thermal conductivity of $4.17 \mathrm{~W} / \mathrm{m}$ $\mathrm{K}$. This result, together with experimental data on the temperature-induced frequency change, indicates that the heating due to the excitation beam should produce a non-negligible change in the Raman frequency, comparable with the observed shifts, but with opposed sign.

In addition to this, a uniform change in the sample temperature may lead to the onset of internal electric fields due to the primary and tertiary pyroelectric effect (arrow $\mathrm{C}$ in Figure 2). The former effect is due to pyroelectric surface charges appearing on the sample surface. Its contribution is estimated to be about one order of magnitude lower than the saturation space charge field value (about $2 \times 10^{6} \mathrm{~V} / \mathrm{m}$ ). The tertiary pyroelectric effect is due to the fact that a temperature gradient along $z$ creates an additional electric field $\boldsymbol{E}_{p y r o}$ so that $\nabla \cdot \boldsymbol{E}_{\text {pyro }}=\left(k / \varepsilon \varepsilon_{0}\right) \partial T / \partial z$, where $k$ is the pyroelectric coefficient. Its contribution is estimated of two orders of magnitude lower than the saturation space charge field. The last indirect thermal contribution is the thermal stress (arrow $D$ in Figure 2), where the temperature gradient creates an elastic strain in the hot region of the sample, which can be shown to be equally negligible.

In summary, the two main contributions to the observed shifts can be individuated in the piezoelectric strain originating from the space charge field and the sample heating caused by light absorption when the sample is absorbing. In the case of samples with limited absorption, the former contribution appears to be preponderant. The following aspect will be further discussed using a simple photorefractivity model.

\section{B. One-center model analysis}

The above reported analysis indicates the photorefractive space charge field as the possible origin of an elastic strain through the piezoelectric effect, just as the same field induces a change in the optical properties through the electro optic effect. Raman spectroscopy is sensitive to strain changes so that the Raman frequency change during time should exhibit some typical features of the photorefractive phenomena. According to experiments performed under applied electric field, $\Delta f(t) \approx s E(t)$, where $s=10^{-7} \mathrm{~cm}^{-1} \frac{\mathrm{m}}{\mathrm{V}}$ was estimated for the $\mathrm{A}_{1}\left[\mathrm{TO}_{4}\right]$ mode. Using this value and assuming for the moment that the space charge field is the sole origin of the observed shifts, we may use our data to obtain the time evolution of the field amplitude in the different samples.

For sake of simplicity, in this first analysis, we will approximate the illumination as uniform inside the spot area, with an average intensity given by the beam power divided by the spot area. Of course this is a crude approximation but allows capturing the essential features of the phenomenon using simple mathematics. In this case, according to the well-known one-center model for photorefractive phenomena in $\mathrm{Fe}: \mathrm{LN},{ }^{6}$ the space charge field should evolve following a saturated exponential law:

$$
E(t)=E_{\text {sat }}\left[1-\exp \left(-\frac{t}{\tau}\right)\right]
$$

The parameter $E_{\text {sat }}$ is proportional to the concentration of $\mathrm{Fe}^{3+}$ ions, ${ }^{16}$ according to the formula

$$
E_{s a t}=\frac{\kappa_{p h} h \nu r}{\mu e}\left[F e^{3+}\right],
$$

where $\kappa_{\mathrm{ph}}$ is the Glass constant, $h \nu$ is the photon energy, $r$ is the recombination rate, $\mu$ is the mobility of light induced charge carriers, and $e$ is the elementary charge. The expected value of the proportionality constant in Eq. (2) is of the order of $10^{-17} \div 10^{-18} \mathrm{Vm}^{2} .^{12}$ On the other hand, the time constant $\tau$ in Eq. (1) is given by

$$
\tau=\frac{\varepsilon \varepsilon_{0}}{\sigma_{P C}},
$$

where $\sigma_{\mathrm{PC}}$ is the material photoconductivity and the dark conductivity of the sample was neglected. Again, from published data, ${ }^{16}$ in a quite large range of experimental parameters and for not too high light intensities $\left(\mathrm{I}<10 \mathrm{GW} / \mathrm{m}^{2}\right)$, the specific photoconductivity value (i.e., the photoconductivity normalized for the intensity and the reduction ratio) is

$$
\frac{\sigma_{P C}}{I} \times \frac{\left[F e^{3+}\right]}{\left[F e^{2+}\right]} \cong 10^{-14} \frac{m}{\mathrm{~V}^{2}} .
$$




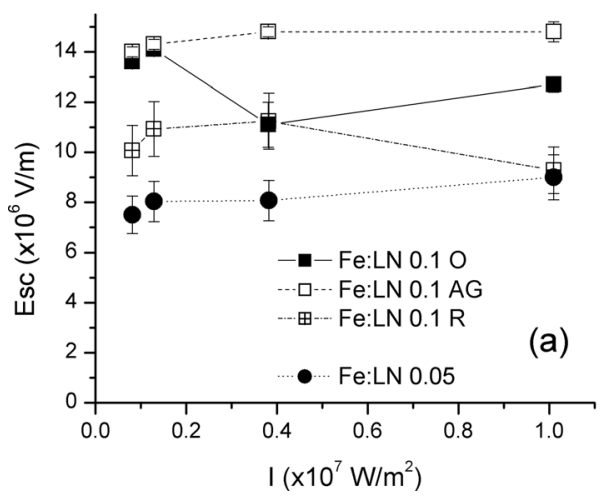

According to this view, the time evolution of the Raman frequency shift of the $\mathrm{A}_{1}\left[\mathrm{TO}_{4}\right]$ line was first converted in the space charge amplitude using the constant $s$ and subsequently fitted using Eqs. (1)-(3) to extract $E_{\text {sat }}$ and $\sigma_{P C}$ as a function of the light intensity for the different samples investigated.

In Figures 4(a) and 4(b), the obtained results for $E_{\text {sat }}$ are reported. The measured values of the saturation space charge field do not show a dependence upon the light intensity, as expected from the one center model. The scattering of the data points at high intensities may be ascribed to the influence of thermal effects and/or to a drift of the experimental conditions when the measurement is particularly long. The saturated space charge field correlates quite well with the total concentration of $\mathrm{Fe}^{3+}$ ions in the different samples (Figure 3(b)), especially when low intensity values are considered. As shown in Figure 3(b), the proportionality constant between the saturation field and the $\left[\mathrm{Fe}^{3+}\right]$ concentration is close to the value found in literature. ${ }^{12}$

The photoconductivity values obtained from the analysis of the Raman data are reported in Figure 5(a). They are proportional to the light intensity and the slope of each line depends on the reduction ratio $\frac{\left[\mathrm{Fe}^{2+}\right]}{\left[\mathrm{Fe}^{3+}\right]}$ of each sample, as expected from Eq. (4). However, the obtained value of the specific photoconductivity is of the order of $10^{-16} \mathrm{~m} / \mathrm{V}^{2}$ (Figure 4(b)), i.e., two orders of magnitude lower than the one expected from Ref. 16 (see Eq. (4)). The contribution due to thermal effects cannot explain this large difference. First of all the magnitude of the thermal contribution is smaller than the one of the photogalvanic field, as explained before. Second, the time constant of thermal phenomena in lithium niobate lies in the seconds range. ${ }^{17}$ If a thermal contribution is assumed, the time dependent shift of the Raman frequencies should be modeled with a double exponential function and the overall characteristic time of the phenomenon would then be some kind of weighted average of the two single characteristic times: even in this case, it should be much smaller than the observed time constants.

On the other hand, the discrepancy is so large that it cannot be due to the approximations involved in our treatment, although coarse. We believe that it can be understood taking into account the poor spatial resolution of the Raman setup. As explained above, in order to have a fast acquisition time for the Raman spectra, the confocal hole was intentionally left wide open, so that the detector integrates signals coming from different regions of the sample. As the speed of the photorefractive phenomena depends on the illumination conditions, weakly illuminated regions produce a Raman signal with a much slower evolution compared to the ones placed in the centre of the beam. The final result is a weighted average of signals and it is likely that the main contribution to the observed phenomena comes precisely from the weakly illuminated regions; in practice, in our experimental conditions, the Raman signal coming from the center of the beam is smaller than the sum of all the other weak signals coming from the whole remaining detection volume. In fact, according to the expected value of the photorefractive time constant (Eqs. (3) and (4)), the Raman signal coming from regions in full illumination close to the center of the beam should evolve about 100 times faster than the ones reported here, so that its detection is practically impossible using our measurement conditions. It is therefore likely that, when a micro-Raman experiment is performed in photorefractive $\mathrm{Fe}: \mathrm{LiNbO}_{3}$ with standard settings (e.g., stronger microscope
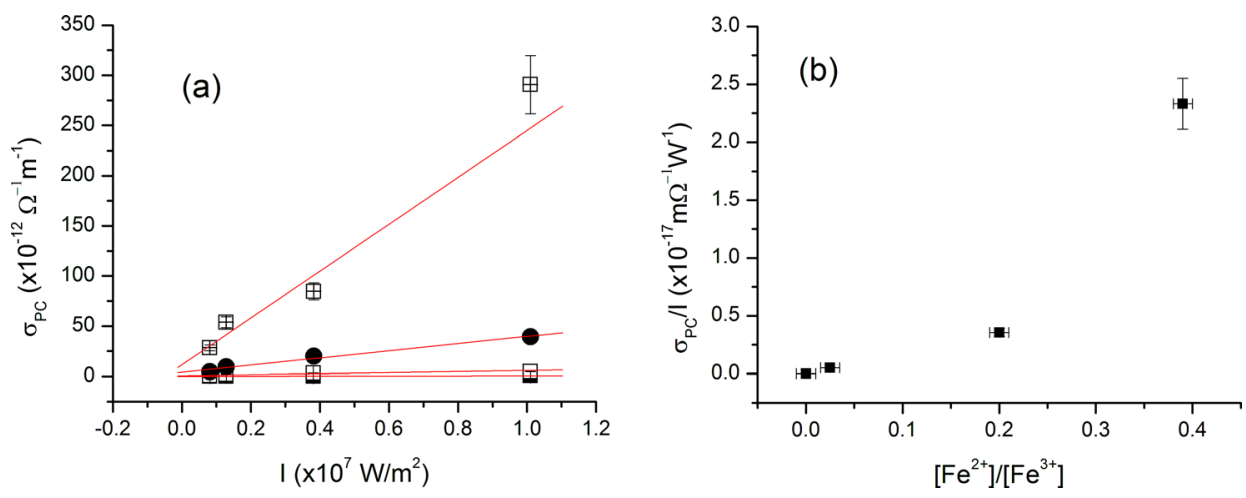

FIG. 5. (a) Photoconductivity versus incident intensity in the different samples, determined by converting the shift in the Raman frequency in space charge field and subsequently fitting with Eqs. (1) and (2). (b) Specific photoconductivity as a function of the $\left[\mathrm{Fe}^{2+}\right] /\left[\mathrm{Fe}^{3+}\right]$ ratio in the different samples. The error bars represent only random errors.

FIG. 4. (a) Saturation value of the space
charge field as a function of the incident intensity determined by converting the charge field and subsequent fitting with Eq. (1). The connecting lines are only a
guide to the eye. (b) Saturated space charge field value versus nominal $\mathrm{Fe}^{3+}$ black line is the theoretical line with a slope of $10^{-18} \mathrm{Vm}^{2}$ between $\mathrm{E}_{\mathrm{sat}}$ and $\left[\mathrm{Fe}^{3+}\right]$ computed from Ref. 12 . 
objectives, smaller pinhole size, etc.) the phenomenon is practically invisible, yet present.

\section{CONCLUSIONS}

In this paper, we showed that under proper experimental conditions, a significant shift in the frequency of the Raman lines can be evidenced in experiments performed on photorefractive Fe:LN. Even if a more detailed analysis is required to quantitatively describe the phenomenon, we believe that the experimental work performed here is sufficient to assess the following points: (1) the frequency shift is principally due to the strain originated by the photorefractive field through the piezoelectric effect, although a minor contribution due to thermal effects may be present; (2) the phenomenon is likely to be always present but hardly detectable because, for typical intensity values, only the evolution of Raman signals coming from the peripheral regions of the illuminated area (normally disregarded in confocal microscope systems) can be followed. One should keep this in mind whenever interested in an absolute measure of the Raman frequency in strongly photorefractive materials such as $\mathrm{Fe}: \mathrm{LiNbO}_{3}$, as the effect can lead to an error as high as $1 \mathrm{~cm}^{-1}$.

Using our setup, we were not able to obtain experimental conditions enabling the detection of the phenomenon using a true confocal arrangement. However, it is not forbidden, in principle, to find such conditions by employing, e.g., other excitation wavelengths or slower materials. If this view is confirmed, we believe that this technique could be used to measure the photorefractive properties of a given sample in a simple fashion and with high spatial resolution. This could be useful to characterize the photorefractive properties of microstructured substrates, such as optical circuits or integrated photorefractive waveguides.

\section{ACKNOWLEDGMENTS}

This work had been partially financed by the "Chip \& CIOP" project of the University of Padua.

${ }^{1}$ I. R. Lewis and H. Edwards, Handbook of Raman Spectroscopy: From the Research Laboratory to the Process Line (Taylor \& Francis, 2001).

${ }^{2}$ Y. Zhang, L. Guilbert, P. Bourson, K. Polgár, and M. D. Fontana, J. Phys.: Condens. Matter 18, 957 (2006).

${ }^{3}$ S. Mignoni, M. D. Fontana, M. Bazzan, M. V. Ciampolillo, A. M. Zaltron, N. Argiolas, and C. Sada, Appl. Phys. B 101, 541 (2010).

${ }^{4}$ R. S. Weis and T. K. Gaylord, Appl. Phys. A 37, 191 (1985).

${ }^{5}$ L. Arizmendi, Phys. Status Solidi A 201, 253 (2004).

${ }^{6} \mathrm{P}$. Gunter and J. P. Huignard, Photorefractive Materials and their Applications 1: Basic Effects (Springer-Verlag, Berlin, 2006), p. 426.

${ }^{7}$ M. Nippus and R. Claus, Z. Naturforsch. Teil A 33, 924 (1978).

${ }^{8}$ R. Mouras, M. D. Fontana, M. Mostefa, and P. Bourson, J. Opt. Soc. Am. B 23, 1867 (2006).

${ }^{9}$ M. V. Ciampolillo, A. Zaltron, M. Bazzan, N. Argiolas, and C. Sada, Appl. Spectrosc. 65, 216 (2011).

${ }^{10}$ A. Ridah, P. Bourson, M. D. Fontana, and G. Malovichko, J. Phys.: Condens. Matter 9, 9687 (1997).

${ }^{11}$ G. Stone, B. Knorr, V. Gopalan, and V. Dierolf, Phys. Rev. B 84, 134303 (2011).

${ }^{12}$ R. Grousson, M. Henry, S. Mallick, and S. L. Xu, J. Appl. Phys. 54, 3012 (1983).

${ }^{13}$ D. Irzhak, D. Roshchupkin, and D. Punegov, Ferroelectrics 351, 163 (2007).

${ }^{14}$ I. D. Wolf, Semicond. Sci. Technol. 11, 139 (1996).

${ }^{15}$ J. Chen and C. Hu, J. Cryst. Growth 158, 289 (1996).

${ }^{16} \mathrm{~T}$. Volk and M. Wöhlecke, Lithium Niobate: Defects, Photorefraction, and Ferroelectric Switching (Springer, Berlin, 2008), p. 264.

${ }^{17}$ S. M. Kostritskii, M. Aillerie, and O. G. Sevostyanov, J. Appl. Phys. 107, 123526 (2010). 